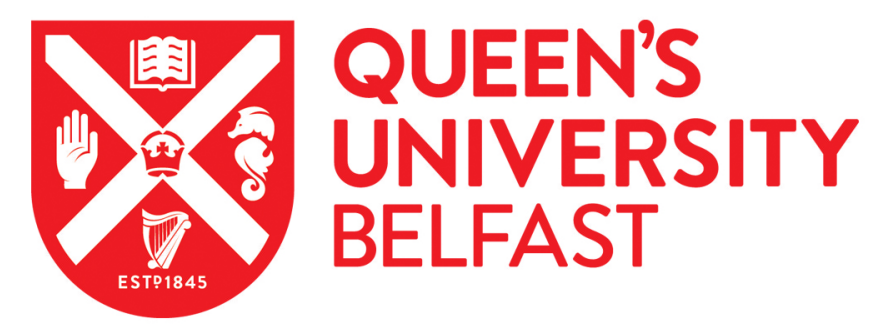

\title{
Repeated MCIK-OFDM with Enhanced Transmit Diversity Under CSI Uncertainty
}

Luong, T. V., Ko, Y., \& Choi, J. (2018). Repeated MCIK-OFDM with Enhanced Transmit Diversity Under CSI Uncertainty. IEEE Transactions on Wireless Communications, 17(6), 4079 - 4088.

https://doi.org/10.1109/TWC.2018.2819997

\section{Published in:}

IEEE Transactions on Wireless Communications

\section{Document Version:}

Peer reviewed version

\section{Queen's University Belfast - Research Portal:}

Link to publication record in Queen's University Belfast Research Portal

Publisher rights

Copyright 2018 IEEE. This work is made available online in accordance with the publisher's policies. Please refer to any applicable terms of use of the publisher.

\section{General rights}

Copyright for the publications made accessible via the Queen's University Belfast Research Portal is retained by the author(s) and / or other copyright owners and it is a condition of accessing these publications that users recognise and abide by the legal requirements associated with these rights.

Take down policy

The Research Portal is Queen's institutional repository that provides access to Queen's research output. Every effort has been made to ensure that content in the Research Portal does not infringe any person's rights, or applicable UK laws. If you discover content in the Research Portal that you believe breaches copyright or violates any law, please contact openaccess@qub.ac.uk. 


\title{
Repeated MCIK-OFDM with Enhanced Transmit Diversity Under CSI Uncertainty
}

\author{
Thien Van Luong, Youngwook Ko, and Jinho Choi
}

\begin{abstract}
This paper investigates the opportunity for a repetition coded Multi-Carrier Index Keying - Orthogonal Frequency Division Multiplexing (MCIK-OFDM), termed as repeated MCIK-OFDM (ReMO), which can provide a superior performance over existing schemes at the same spectral efficiency. Unlike the classical scheme, the proposed scheme activates a subset of sub-carriers and modulates them with the same $M$ ary data symbol, while additional information is conveyed by the active sub-carrier indices. This approach not only provides the frequency diversity gains in the $M$-ary symbol detection but also improves the index detection, leading to a considerable improvement in the transmit diversity. For performance analysis, we derive tight, closed-form expressions for the symbol error probability (SEP) and the bit error rate (BER), under both the perfect and imperfect channel state information (CSI). These expressions provide an insight into the achievable performance gains, system designs and impacts of various CSI conditions. Finally, simulation results are given to illustrate the superior performance achieved by our scheme over existing schemes under different CSI uncertainties.
\end{abstract}

Index Terms-MICK-OFDM, OFDM-IM, index modulation, symbol error probability, frequency diversity, transmit diversity.

\section{INTRODUCTION}

Multi-Carrier Index Keying - Orthogonal Frequency Division Multiplexing (MCIK-OFDM) also known as OFDM with Index Modulation (OFDM-IM) [1], [2], has recently emerged as a promising multi-carrier technique, which can provide higher spectral, energy efficiency and reliability than conventional OFDM. In MCIK-OFDM, only a subset of sub-carriers are activated to convey data bits via both the $M$-ary symbols and the indices of active sub-carriers. This allows MCIK-OFDM to reduce the complexity of transceiver structure since less modulators and demodulators are required. Moreover, MCIK-OFDM is able to offer an interesting tradeoff between reliability and spectral efficiency (SE) by adjusting the number of active subcarriers [2]. Thus, MCIK-OFDM is considered as a competitive candidate for machine type communications (MTC) [3], which require a low-cost, lowpower, low-complexity and highly reliable system.

A variety of MCIK-OFDM concepts have been studied over the past few years [4]. The MCIK was initially introduced in [1], in which only half of sub-carriers are activated. Then, the number of active sub-carriers is relaxed in [2]. In [5], a tight upper bound on the bit error rate (BER) is derived. Then, the

Thien Van Luong and Youngwook Ko are with the Electronics, Communications and Information Technology Institute, Queen's University Belfast, Belfast, BT3 9DT United Kingdom, and Jinho Choi is with the School of Electrical Engineering and Computer Science, Gwangju Institute of Science and Technology (GIST), Gwangju, 61005, Korea. achievable data rate of MCIK-OFDM is investigated in [6]. To increase the SE, in [7], the IM is applied to both the inphase and quadrature components to double the number of index bits, which results in a scheme named as OFDM-IMI/Q. A generalized MCIK-OFDM is presented in [8], where the number of active sub-carriers is no longer fixed. Dualmode OFDM (DM-OFDM) is proposed in [9], which exploits inactive sub-carriers to carry additional data. Recently, the novel frameworks to derive the symbol error probability (SEP) and the BER of MCIK-OFDM under channel state information (CSI) uncertainty can be found in [10], [11], respectively.

As for the diversity issue, in [12], the subcarrier-level interleaving technique is introduced to OFDM-IM, which can provide the better BER over the classical schemes, but not increase the diversity order of systems. In [13], the coordinate interleaved OFDM-IM (CI-OFDM-IM) that transmits real and imaginary parts of complex symbols over different sub-carriers to obtain transmit diversity is presented. In [14], OFDM-IM with transmit diversity (OFDM-IM-TD) is proposed, which employs multiple signal constellations to transmit the same data bits via both active and silent sub-carriers. The linear constellation precoding (LCP) is applied to OFDM-IM-I/Q in [15], to achieve the diversity order of two at the cost of complexity in the optimization of the precoding matrix. In [16], coded OFDM-IM is proposed to achieve a diversity gain for index detection. Several studies that extend the MCIK concept to multiple-input multiple-output (MIMO) system are presented in [17]-[21].

It is noteworthy that such complicated transceiver designs may not be suitable for low-complexity MTC applications. This motivates us to develop a simple transmit diversity structure that can improve reliability at no cost of data rate. Our previous work [22] recently proposed a frequency diversity for MCIK-OFDM to enhance the symbol error rate. However, this lacks theoretical analysis on the achievable diversity and power gains, system designs as well as an error floor, especially with uncertain channel information. To the best of our knowledge, the potential of using the frequency diversity for MCIK concepts has been little explored.

In this paper, considering MCIK-OFDM under MTC scenarios, we propose a new IM scheme that not only has a simple design, but also significantly enhances the error performance, with various channel uncertainties. Main contributions are summarized as follows.

- A repeated MCIK-OFDM termed as ReMO is proposed, which combines MCIK with frequency diversity to modulate the same $M$-ary symbol over all active sub-carriers. This simple but effective method allows to not only 
provide the frequency diversity gain in the $M$-ary symbol detection, but also enhance the index detection performance.

- We derive closed-form expressions for both the average pairwise index error probability (PEP) and SEP. Then, based on this, the generalized expression for the BER is provided. These expressions reveal that the proposed scheme can achieve the diversity order of two, which is twice higher than the classical scheme. We also gain an insight into impacts of parameters such as the modulation size and the number of active sub-carriers on the SEP, which result in the theoretical guideline of designing systems to achieve the best SEP.

- We then extend the proposed scheme to the imperfect CSI. The expressions for the average PEP and SEP taking into account various CSI conditions are derived, which allow to investigate impacts of various CSI uncertainties on the SEP such as the fixed and minimum mean square error (MMSE)-based variable CSI, as well as an error floor and the performance loss.

- Simulation results are presented to verify the performance and the theoretical analysis. Specifically, our proposed scheme without any additional techniques such as CI, I/Q, LPC and multiple constellations still achieves a superior performance over existing schemes under different CSI uncertainties, even at the same data rate. In addition, derived theoretical expressions are accurate in a range of signal-to-noise ratio (SNR) regions.

The rest of this paper is organised as follows. Section II presents the system model. The PEP and SEP performance analysis is provided Section III. In Section IV, the impact of CSI uncertainty on the average SEP is investigated. The simulation results are given in Section VI. Finally, Section V concludes our work.

Notation: $C($,$) and \lfloor$.$\rfloor denote the binomial coefficient and$ the floor function, respectively. Column vectors and matrices are presented by bold lowercase and capital letters, respectively. $\mathbb{E}\{$.$\} and \mathcal{M}($.$) denote the expectation operation and$ the moment generating function (MGF), respectively. $(.)^{T}$ is the complex conjugation and $\mathcal{C N}($,$) denotes the complex$ Gaussian distribution, respectively. $\Theta($.$) is Big-Theta notation.$

\section{SYSTEM MODEL}

Consider a multi-carrier system with $N_{c}=G N$ subcarriers, consisting of $G$ blocks of $N$ sub-carriers. Like classical MCIK-OFDM, only $K$ out of $N$ sub-carriers in each block are activated to carry data and remaining inactive ones are zero padded. Unlike conventional scheme, which delivers distinct $M$-ary symbols over $K$ active sub-carriers, we propose to repeatedly use the same $M$-ary symbol $s$ in modulating $K \geq 2$ active sub-carriers. This scheme is referred to as repeated MCIK-OFDM (ReMO) throughout the paper. The proposed approach can exploit the diversity gain in both the frequency and index domains at the cost of degraded SE due to the data symbol repetition. Since such a mapping is independently applied to every block, without loss of generality, hereinafter, we address only one block.
In every transmission of each block, incoming $p_{1}+p_{2}$ bits are split into two groups at the transmitter. The first $p_{1}$ bits are mapped to an index symbol, which corresponds to a combination of $K$ active sub-carriers whose indices are in a set of $\theta$, i.e., $\theta=\left\{\alpha_{1}, . ., \alpha_{K}\right\}$, where $\alpha_{k} \in\{1, \ldots, N\}$. Here, the index symbol can be defined by a vector as $\boldsymbol{\lambda}=\left[\beta_{1}, \ldots, \beta_{N}\right]^{T}$, where $\beta_{i}=1$ if $i \in \theta$ and $\beta_{i}=0$ if $i \notin \theta$. For given $N$ and $K$, let $\Phi$ be the set of index symbols in total and its cardinality is given by $m_{1}=|\Phi|=2^{\left\lfloor\log _{2} C(K, N)\right\rfloor}$. This leads to $p_{1}=\left\lfloor\log _{2} C(N, K)\right\rfloor$. In addition, the combinatorial method [2] is used to generate the $m_{1}$ index symbols for our scheme. The remaining $p_{2}$ bits $\left(p_{2}=\log _{2} M\right)$ are modulated by one $M$-ary symbol $s$. Accordingly, the transmitted codeword uses index modulation and frequency diversity to combine index symbol $\boldsymbol{\lambda}$ and the $M$-ary symbol $s$, as $\mathbf{x}=\boldsymbol{\lambda} s$.

The received signal $\boldsymbol{y}$ in the frequency domain is given as

$$
\mathbf{y}=\mathbf{H} \boldsymbol{\lambda} s+\mathbf{n},
$$

where $\mathbf{H}=\operatorname{diag}\{h(1), \ldots, h(N)\}$ denotes the channel matrix, where $h(\alpha)$ is a flat Rayleigh fading channel of sub-carrier $\alpha$ and $h(\alpha) \sim \mathcal{C N}(0,1)$, and $\mathbf{n}=[n(1), . ., n(N)]^{T}$ is the additive Gaussian noise vector, $n(\alpha) \sim \mathcal{C N}\left(0, N_{0}\right)$. For the power constraint, we assume that $\mathbb{E}\left\{\left|s^{2}\right|\right\}=\varphi E_{s}$, where $E_{s}$ and $\varphi=N / K$ are the average transmit power per sub-carrier and the power allocation coefficient, respectively. Hence, the average SNR per active sub-carrier is given by $\bar{\gamma}=\varphi E_{s} / N_{0}$.

Finally, to recover the transmitted signal, the maximum likelihood (ML) detector is employed to estimate $\hat{\boldsymbol{x}}$ as follows

$$
\hat{\mathbf{x}}=(\hat{\boldsymbol{\lambda}}, \hat{s})=\arg \min _{\boldsymbol{\lambda}, s}\|\mathbf{y}-\mathbf{H} \boldsymbol{\lambda} s\|^{2} .
$$

The ML complexity in terms of complex multiplications is $\sim \mathcal{O}\left(m_{1} M\right)$, which increases linearly with $M$.

\section{ERRor PERFORMANCE ANALYSIS}

We analytically evaluate the SEP of the proposed scheme with the ML detection. For the mathematical tractability in the derivation, we assume the CSI is perfectly known at the receiver. The analysis for the imperfect CSI case will be presented in the next section.

Let us define the SEP as the ratio of the number of symbols in error to the total of the transmitted index and $M$-ary symbols. This consists of two symbol error types, namely the index symbol error and the $M$-ary symbol error.

\section{A. Pairwise Index Error Probability}

With the index symbol error, we first consider the index detection; the well-known conditional pairwise index-detection error probability (PEP) of the ML detection can be used to represent the error event that the transmitted $i$-th index vector is incorrectly detected as $j$-th index vector. Assume that $s$ is an $M$-ary PSK symbol with the envelope $|s|=\sqrt{\varphi E_{s}}$. The corresponding PEP can be given by

$$
P\left(\boldsymbol{\lambda}_{i} \rightarrow \boldsymbol{\lambda}_{j}\right)=Q\left(\sqrt{\frac{\varphi E_{s}\left\|\mathbf{H} \boldsymbol{\lambda}_{i}-\mathbf{H} \boldsymbol{\lambda}_{j}\right\|^{2}}{2 N_{0}}}\right),
$$


where $\boldsymbol{\lambda}_{i}$ and $\boldsymbol{\lambda}_{j}$ denote the transmitted index vector and the decoded index vector, respectively, and $Q(x)=$ $\frac{1}{2 \pi} \int_{x}^{\infty} \exp \left(-\frac{t^{2}}{2}\right) \mathrm{d} t$ is the Gaussian tail probability [23].

Let $\boldsymbol{\lambda}_{j}$ and $\boldsymbol{\lambda}_{i}$ have a Hamming distance of $2 D$. Then, we have $\left\|\mathbf{H} \boldsymbol{\lambda}_{i}-\mathbf{H} \boldsymbol{\lambda}_{j}\right\|^{2}=\left\|\mathbf{H} \boldsymbol{\lambda}_{e}\right\|^{2}$, where $\boldsymbol{\lambda}_{e}=\boldsymbol{\lambda}_{i}-\boldsymbol{\lambda}_{j}$ is a zero vector except $2 D$ entities. Thus, the PEP in (3) can be represented as

$$
P\left(\boldsymbol{\lambda}_{i} \rightarrow \boldsymbol{\lambda}_{j}\right)=Q\left(\sqrt{\frac{\sum_{d=1}^{D}\left(\gamma_{\alpha_{d}}+\gamma_{\tilde{\alpha}_{d}}\right)}{2}}\right)
$$

where $\alpha_{d} \in \theta_{i}$ and $\tilde{\alpha}_{d} \in \theta_{j}$ such that $\alpha_{d}, \tilde{\alpha}_{d} \notin \theta_{i} \cap \theta_{j}$, and $\gamma_{\alpha}=\bar{\gamma}|h(\alpha)|^{2}$ is the instantaneous SNR of sub-carrier $\alpha$.

Using the union bound, the probability of the index misdetetion of $\boldsymbol{\lambda}_{i}$ can be obtained as

$$
P_{i} \leq \sum_{j=1, j \neq i}^{m_{1}} P\left(\boldsymbol{\lambda}_{i} \rightarrow \boldsymbol{\lambda}_{j}\right)
$$

thus, the instantaneous PEP can be expressed as

$$
P_{I}=\frac{1}{m_{1}} \sum_{i=1}^{m_{1}} P_{i} \leq \frac{1}{m_{1}} \sum_{i=1}^{m_{1}} \sum_{j=1, j \neq i}^{m_{1}} P\left(\boldsymbol{\lambda}_{i} \rightarrow \boldsymbol{\lambda}_{j}\right) .
$$

Denote by $\Omega_{i}$ the set of indices $j(\neq i)$ such that $\boldsymbol{\lambda}_{j}$ is satisfying the Hamming distance of 2 with $\boldsymbol{\lambda}_{i}$, i.e., $D=1$ in (4). Denote by $\eta_{i}$ the cardinality of $\Omega_{i}$, i.e., $\eta_{i}=\left|\Omega_{i}\right|$. Based on (4) and (5), notice that $P_{i}$ is dominated by the following terms $P\left(\boldsymbol{\lambda}_{i} \rightarrow \boldsymbol{\lambda}_{j} \mid j \in \Omega_{i}\right)$, i.e., $\sum_{j \neq i=1}^{m_{1}} P\left(\boldsymbol{\lambda}_{i} \rightarrow \boldsymbol{\lambda}_{j}\right) \approx$ $\sum_{j \in \Omega_{i}} P\left(\boldsymbol{\lambda}_{i} \rightarrow \boldsymbol{\lambda}_{j}\right)$. Hence, (6) can be approximated by

$$
P_{I} \approx \frac{1}{m_{1}} \sum_{i=1}^{m_{1}} \sum_{j \in \Omega_{i}} P\left(\boldsymbol{\lambda}_{i} \rightarrow \boldsymbol{\lambda}_{j}\right)=\frac{1}{m_{1}} \sum_{i=1}^{m_{1}} \sum_{j \in \Omega_{i}} P(\alpha \rightarrow \tilde{\alpha}),
$$

where due to $D=1, P(\alpha \rightarrow \tilde{\alpha})=Q\left(\sqrt{\frac{\gamma_{\alpha}+\gamma_{\tilde{\alpha}}}{2}}\right)$ is the simplified PEP of incorrectly detecting an active sub-carrier $\alpha \in \theta_{i}$ as an inactive sub-carrier $\tilde{\alpha} \in \theta_{j}$, where $\theta_{i}, \theta_{j}$ are the corresponding index sets of $\boldsymbol{\lambda}_{i}, \boldsymbol{\lambda}_{j}$, respectively.

The following theorem provides a closed-form expression for the average PEP, when the perfect CSI is used.

Theorem 1. The repetition coded MCIK-OFDM under perfect CSI ensures that the average PEP is achieved as

$$
\bar{P}_{I} \approx \frac{\Psi}{12}\left[\frac{16}{(4+\bar{\gamma})^{2}}+\frac{27}{(3+\bar{\gamma})^{2}}\right]
$$

where $\Psi=\sum_{i=1}^{m_{1}} \eta_{i} / m_{1}$

Proof: See Appendix A.

It can be seen from (8) that the average PEP depends on $N, K$ through $\bar{\gamma}$ and $m_{1}$. Interestingly, notice that $\bar{P}_{I}$ is not affected by the PSK modulation size $M$. Moreover, for given $N$ and $K$, the PEP is influenced by the design of the index symbols $\boldsymbol{\lambda}$ via the term of $\sum_{i=1}^{m_{1}} \eta_{i}$ in $\Psi$. The achievable diversity order is two, i.e., $-\lim _{\bar{\gamma} \rightarrow \infty}\left[\log \left(\bar{P}_{I}\right) / \log (\bar{\gamma})\right]=2$.

\section{B. Symbol Error Probability (SEP)}

A symbol error event occurs if either $\hat{\boldsymbol{\lambda}} \neq \boldsymbol{\lambda}$ or $\hat{s} \neq s$. With each transmission of codeword $\boldsymbol{x}$ consisting of two different symbols ( $\boldsymbol{\lambda}$ and $s$ ), the number of potential symbols in error can be 0,1 or 2 . As a result, the instantaneous SEP and its average can be approximated, respectively by

$$
\begin{aligned}
P_{s} \approx \frac{1}{m_{1}} \sum_{i=1}^{m_{1}} \frac{1}{2} & {\left[P_{M}+\sum_{j \in \Omega_{i}} P(\alpha \rightarrow \tilde{\alpha})\right], } \\
\bar{P}_{s} & \approx \frac{\bar{P}_{I}+\bar{P}_{M}}{2},
\end{aligned}
$$

where $P_{M}$ is the instantaneous SEP of $M$-ary PSK symbols, provided that the detection of active sub-carrier indices $\theta_{i}$ is correct, and $\bar{P}_{M}$ denotes its average.

The following theorem provides a closed-form expression for the average SEP, when the perfect CSI is used.

Theorem 2. The repeated MCIK-OFDM under perfect CSI can yields the tight, approximate average SEP as follows:

$$
\begin{aligned}
\bar{P}_{s} & \approx \frac{\Psi}{24}\left[\frac{16}{(4+\bar{\gamma})^{2}}+\frac{27}{(3+\bar{\gamma})^{2}}\right] \\
& +\frac{1}{12}\left[\frac{1}{(1+\rho \bar{\gamma})^{K}}+\frac{3}{\left(1+\frac{4 \rho \bar{\gamma}}{3}\right)^{K}}\right] .
\end{aligned}
$$

Proof: See Appendix B.

It is seen from (11) that at high SNRs, the average SEP is determined dominantly by the first two terms, which relies on the accuracy of the index detection, as $K$ grows. This is because at $K>2$, the achievable diversity order in (11) is always limited by two. This is the diversity order exactly attained in the index domain. Notice that MCIK-OFDM achieves unit diversity order [10]. Thus, over the classical scheme, attaining diversity order twice higher, the proposed scheme ensures to significantly enhance the SEP performance.

Notice from [2] that authors suggested transmitting data via the active indices only to attain the diversity order of two. However, this approach is not effective and even wasteful in terms of both spectral efficiency and reliability, especially when $K>1$. This is because the index detection errors does not depends on the use of the $M$-ary modulation, i.e., $M$, according to (8). Hence, the ReMO appears to effectively address such the wastefulness of the approach in [2].

Moreover, compared to MCIK-OFDM, the repeated code in proposed scheme is to mainly improve the diversity gain in the $M$-ary symbol detection, not in the index detection performance. This can be observed via the rule of $K$ in (11). Such new insight into the SEP provides that the design of various MCIK-OFDM concepts can better perform, when balancing diversity gains between the index symbol and $M$-ary symbol detections. Particularly, larger $K$ makes diversity gains achieved in the index and $M$-ary domains more unbalanced, which may reduce the overall performance of the ReMO. Thus, in general, smaller $K$ would be preferred.

Remark 1 (Expression of BER): Following the approach of deriving the BER of MCIK-OFDM in [11], we can obtain the approximate expression for the BER of ReMO as follows 


$$
P_{b} \approx \frac{p_{1} \bar{P}_{I} / 2+\bar{P}_{M}}{p_{1}+p_{2}},
$$

where $\bar{P}_{I}$ and $\bar{P}_{M}$ are given in (8) and (30), respectively. Interestingly, the theoretical BER in (12) is accurate in a wide range of SNRs, which will be validated via simulation results.

\section{Asymptotic Analysis}

The $\bar{P}_{s}$ in (11) can be asymptotically approximated, at high SNRs, to

$$
\begin{aligned}
\bar{P}_{s} & \approx \frac{43 K^{2}}{24 N^{2}}\left(\Psi+\frac{\vartheta}{8 \rho^{2}}\right)\left(\frac{1}{\gamma_{0}^{2}}\right) \\
& =\Theta\left(\left(c_{p} \gamma_{0}\right)^{-2}\right),
\end{aligned}
$$

where $\vartheta=1$ and 0 for $K=2$ and $K>2$, respectively, $\gamma_{0}$ denotes the average SNR per sub-carrier, i.e., $\gamma_{0}=E_{s} / N_{0}$, and the coding gain $c_{p}$ given as

$$
c_{p}=\frac{N}{K \sqrt{\frac{43}{24}\left(\Psi+\frac{\vartheta}{8 \rho^{2}}\right)}} .
$$

Based on this, we gain insights into behaviors of the SEP as well as the system designs in the following remarks.

Remark 2. It can be seen from (13) that for $K>2$, we have $\bar{P}_{s} \approx \bar{P}_{I} / 2$ at high SNRs. While for $K=2$, we obtain $\bar{P}_{s} \approx \bar{P}_{I} / 2$ if $M$ is small $(M=2,4,8)$, and $\bar{P}_{s} \approx \bar{P}_{M} / 2$ if $M$ is large $(M \geq 16)$. Hence, for low-throughput transmission with a small $M$, the SEP at high SNRs is mainly affected by the index detection rather than the $M$-ary symbol detection.

Remark 3. For given $N$, the scheme with small $M(M \leq 8)$ achieves the better SEP as $K$ is smaller. As a result, $K=2$ provides the best performance. By contrast, at large $M(M \geq$ 16), larger $K$ is preferred to minimize the SEP due to the dominance of the $M$-ary detection errors when $M$ is large. Particularly, larger $K$ results in higher diversity order of $\bar{P}_{s}$.

\section{ERror Performance Under CSI Uncertainty}

For practical systems, we consider in the presence of CSI uncertainty at the receiver. For this, the ML receiver uses the estimate of the channel matrix $\hat{\mathbf{H}}=\operatorname{diag}\{\hat{h}(1), \ldots, \hat{h}(N)\}$ rather than the true one $\mathbf{H}$ in (2) to decode the transmitted signal. Assume that $\hat{\mathbf{H}}$ satisfies [10]

$$
\mathbf{H}=\hat{\mathbf{H}}+\mathbf{E},
$$

where $\mathbf{E}=\operatorname{diag}\{e(1), \ldots, e(N)\}$ is the channel estimation error, whose elements satisfies $e(\alpha) \sim \mathcal{C N}\left(0, \epsilon^{2}\right)$, where $\epsilon^{2}$ denotes the error variance and $\epsilon^{2} \in[0,1)$, and $\hat{h}(\alpha) \sim$ $\mathcal{C N}\left(0,1-\epsilon^{2}\right)$. Hence, for each sub-channel, we have

$$
h(\alpha)=\hat{h}(\alpha)+e(\alpha) .
$$

We now analyze the impact of the channel estimation error $\epsilon^{2}$ on the PEP and thus on the SEP of systems.

\section{A. PEP under CSI Uncertainty}

Similar to (3), we derive the average PEP with CSI uncertainty. Using $\hat{\mathbf{H}}$ in (15), the received signal in (1) can be rewritten as $\boldsymbol{y}=\hat{\mathbf{H}} \boldsymbol{\lambda}_{i} s+\tilde{\mathbf{n}}$, where $\tilde{\mathbf{n}}=\mathbf{E} \boldsymbol{\lambda}_{i} s+\mathbf{n}$. The elements of $\tilde{\mathbf{n}}$ are given by $\tilde{n}(\alpha)=n(\alpha) \sim \mathcal{C N}\left(0, N_{0}\right)$ for $\alpha \in \theta_{i}$ and $\tilde{n}(\alpha)=e(\alpha) s+n(\alpha) \sim \mathcal{C N}\left(0,\left(1+\bar{\gamma} \epsilon^{2}\right) N_{0}\right)$ for $\alpha \notin \theta_{i}$. Thus, conditioned on $\hat{\mathbf{H}}$, the PEP that the index symbol $\boldsymbol{\lambda}_{i}$ is incorrectly detected as $\boldsymbol{\lambda}_{j} \neq \boldsymbol{\lambda}_{i}$, can be

$$
\begin{aligned}
P\left(\boldsymbol{\lambda}_{i} \rightarrow \boldsymbol{\lambda}_{j}\right) & =P\left\{\left\|\mathbf{y}-\hat{\mathbf{H}} \boldsymbol{\lambda}_{i} s\right\|^{2}>\left\|\mathbf{y}-\hat{\mathbf{H}} \boldsymbol{\lambda}_{j} s\right\|^{2}\right\} \\
& =P\left\{\|\tilde{\mathbf{n}}\|^{2}>\left\|\hat{\mathbf{H}} \boldsymbol{\lambda}_{i j} s+\tilde{\mathbf{n}}\right\|^{2}\right\} \\
& =P\left\{-2 \Re\left\{\tilde{\mathbf{n}}{ }^{H} \hat{\mathbf{H}} \boldsymbol{\lambda}_{i j} s\right\}>\left\|\hat{\mathbf{H}} \boldsymbol{\lambda}_{i, j} s\right\|^{2}\right\},
\end{aligned}
$$

where $\boldsymbol{\lambda}_{i j}=\boldsymbol{\lambda}_{i}-\boldsymbol{\lambda}_{j}$.

Let us define $\theta_{i j}^{i}=\left\{\alpha \mid \alpha \in \theta_{i}, \alpha \notin \theta_{j}\right\}$ and $\theta_{i j}=\theta_{i j}^{i} \cup$ $\theta_{j i}^{j}$ for $i \neq j=1, \ldots, m_{1}$. Based on (17), notice that $-\mathfrak{R}\left\{\tilde{\mathbf{n}}^{H} \hat{\mathbf{H}} \boldsymbol{\lambda}_{i, j} s\right\}$ is a real Gaussian variable with zero mean and variance $N_{0}\left[\sum_{\alpha \in \theta_{j i}^{j}} \hat{\gamma}_{\alpha}+\left(1+\bar{\gamma} \epsilon^{2}\right) \sum_{\alpha \in \theta_{i j}^{i}} \hat{\gamma}_{\alpha}\right]$, and $\left\|\hat{\mathbf{H}} \boldsymbol{\lambda}_{i j} s\right\|^{2}=N_{0} \sum_{\alpha \in \theta_{i j}} \hat{\gamma}_{\alpha}$, where $\hat{\gamma}_{\alpha}=\bar{\gamma}|\hat{h}(\alpha)|^{2}$ for $\alpha=1, \ldots, N$. Therefore, using this observation and some mathematical manipulation from (17), we can attain

$$
\begin{aligned}
P\left(\boldsymbol{\lambda}_{i} \rightarrow \boldsymbol{\lambda}_{j}\right) & =Q\left[\sqrt{\frac{\sum_{\alpha \in \theta_{i j}} \hat{\gamma}_{\alpha}}{2\left(1+\frac{\sum_{\alpha \in \theta_{i j}^{i}} \hat{\gamma}_{\alpha}}{\sum_{\alpha \in \theta_{i j}} \hat{\gamma}_{\alpha}} \bar{\gamma} \epsilon^{2}\right)}}\right] \\
& \approx Q\left[\sqrt{\frac{\sum_{\alpha \in \theta_{i j}} \hat{\gamma}_{\alpha}}{2+\bar{\gamma} \epsilon^{2}}}\right],
\end{aligned}
$$

where we take an approximation of $\sum_{\alpha \in \theta_{i j}^{i}} \hat{\gamma}_{\alpha} / \sum_{\alpha \in \theta_{i j}} \hat{\gamma}_{\alpha} \approx$ $1 / 2$ to simplify the formulation of the conditional PEP.

As seen from (18) that the instantaneous PEP in (6) is dominated by the conditional PEPs $P\left(\boldsymbol{\lambda}_{i} \rightarrow \boldsymbol{\lambda}_{j}\right)$, that satisfy $\left|\theta_{i j}\right|$ is minimum, i.e., $\left|\theta_{i j}\right|=2$. This is due to the fact that $\left|\theta_{i j}\right|=2 D \geq 2$, where $D$ is the Hamming distance between $\boldsymbol{\lambda}_{i}$ and $\boldsymbol{\lambda}_{j}$. Hence, denote the set $\Omega_{i}=\left\{j|| \theta_{i j} \mid=2\right\}$ and its cardinality $\eta_{i}=\left|\Omega_{i}\right|$, we obtain the instantaneous PEP under CSI uncertainty, using (6) as

$$
P_{I} \approx \frac{1}{m_{1}} \sum_{i=1}^{m_{1}} \sum_{j \in \Omega_{i}} Q\left[\sqrt{\frac{\hat{\gamma}_{\alpha}+\hat{\gamma}_{\tilde{\alpha}}}{2+\bar{\gamma} \epsilon^{2}}}\right],
$$

where $\alpha \in \theta_{i j}^{i}$ and $\tilde{\alpha} \in \theta_{j i}^{j}$.

The average PEP can be extended to the uncertain CSI case, as given in the following theorem.

Theorem 3. The closed-form expression for the average PEP of the repetition coded MCIK-OFDM in the presence of CSI uncertainty is given by

$$
\bar{P}_{I} \approx \frac{\Psi}{12}\left\{\frac{1}{\left[1+\frac{\bar{\gamma}\left(1-\epsilon^{2}\right)}{4+2 \bar{\gamma} \epsilon^{2}}\right]^{2}}+\frac{3}{\left[1+\frac{2 \bar{\gamma}\left(1-\epsilon^{2}\right)}{6+3 \bar{\gamma} \epsilon^{2}}\right]^{2}}\right\} .
$$


Proof: See Appendix C.

It can be seen that (20) is the generalized expression for (8), as $\epsilon^{2}=0$. Particularly, for $\epsilon^{2}>0$ the average PEP is higher than the performance with the perfect CSI $\left(\epsilon^{2}=0\right)$.

\section{B. SEP under CSI Uncertainty}

To derive the average SEP under CSI uncertainty, we first address the average SEP of the $M$-ary symbols under CSI imperfection as shown in the following lemma.

Lemma 1. In the repeated MCIK-OFDM with uncertain $C S I$, the average SEP of the M-ary PSK conditioned on the correct detection of active sub-carrier indices is given by

$$
\bar{P}_{M} \approx \frac{1}{6}\left\{\frac{1}{\left[1+\frac{\left(1-\epsilon^{2}\right) \bar{\gamma} \rho}{1+\bar{\gamma} \epsilon^{2}}\right]^{K}}+\frac{3}{\left[1+\frac{4\left(1-\epsilon^{2}\right) \bar{\gamma} \rho}{3+3 \bar{\gamma} \epsilon^{2}}\right]^{K}}\right\},
$$

where $\rho=\sin ^{2}(\pi / M)$.

Proof: See Appendix D.

The following theorem provides the average SEP in the presence of CSI uncertainty.

Theorem 4. The repetition coded MCIK-OFDM with uncertain CSI ensures that the following average SEP is attained

$$
\begin{aligned}
\bar{P}_{s} & \approx \frac{\Psi}{24}\left\{\frac{1}{\left[1+\frac{\bar{\gamma}\left(1-\epsilon^{2}\right)}{4+2 \bar{\gamma} \epsilon^{2}}\right]^{2}}+\frac{3}{\left[1+\frac{2 \bar{\gamma}\left(1-\epsilon^{2}\right)}{6+3 \bar{\gamma} \epsilon^{2}}\right]^{2}}\right\} \\
& +\frac{1}{12}\left\{\frac{1}{\left[1+\frac{\left(1-\epsilon^{2}\right) \bar{\gamma} \rho}{1+\bar{\gamma} \epsilon^{2}}\right]^{K}}+\frac{3}{\left[1+\frac{4\left(1-\epsilon^{2}\right) \bar{\gamma} \rho}{3+3 \bar{\gamma} \epsilon^{2}}\right]^{K}}\right\} .
\end{aligned}
$$

Proof: Using Theorem 3 and Lemma 1, and based on (10), we obtain (22). This concludes the proof.

It is shown from (22) that the error variance $\epsilon^{2}(>0)$ causes a degradation in the SEP performance compared to the perfect CSI case. In addition, the BER under imperfect CSI can be obtained by substituting (20) and (21) into (12).

\section{Impact of CSI Uncertainty and Error Floor}

We now present the asymptotic analysis on the SEP of the proposed scheme with $K>1$, in order to evaluate impacts of various CSI uncertainties. For this, we consider two imperfect CSI conditions including fixed and MMSE-based variable CSI.

1) Error Floor by Fixed CSI Uncertainty $\left(\epsilon^{2}>0\right)$ : For fixed $\epsilon^{2}>0$, in high SNR regions, an error floor of $\bar{P}_{s}$ in (22) can be asymptotically approximated by

$$
\begin{aligned}
\bar{P}_{s} & \approx \frac{\Psi}{24}\left[\frac{1}{\left(1+\frac{1-\epsilon^{2}}{2 \epsilon^{2}}\right)^{2}}+\frac{3}{\left(1+\frac{2-2 \epsilon^{2}}{3 \epsilon^{2}}\right)^{2}}\right] \\
& +\frac{1}{12}\left\{\frac{1}{\left[1+\frac{\left(1-\epsilon^{2}\right) \rho}{\epsilon^{2}}\right]^{K}}+\frac{3}{\left[1+\frac{4\left(1-\epsilon^{2}\right) \rho}{3 \epsilon^{2}}\right]^{K}}\right\} .
\end{aligned}
$$

As shown from (23), at large $\bar{\gamma}$, the SEP is no longer a function of the SNR, that is, as the SNR grows, the average SEP relies on only $N, K$ and $\epsilon^{2}$. This means there exists an error floor of the SEP, which gets higher as increasing $\epsilon^{2}$.

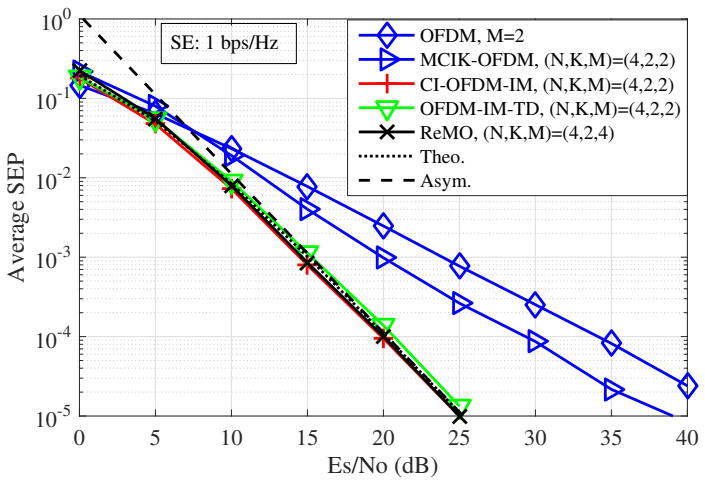

Fig. 1. Average SEP comparison among classical OFDM with $M=$ 2, MCIK-OFDM, CI-OFDM-IM and OFDM-IM-TD with $(N, K, M)=$ $(4,2,2)$, and ReMO with $(N, K, M)=(4,2,4)$, at the $\mathrm{SE}$ of $1 \mathrm{bps} / \mathrm{Hz}$.

Remark 4. It is worth noting from (23) that for given $N$ and $\epsilon^{2}$, larger $K$ and smaller $M$ provide the lower error floor. Moreover, for given $K$ and $\epsilon^{2}$, smaller $N$ offers the lower error floor. These observations would be useful to choose the system parameters that minimize the SEP under fixed CSI condition.

2) MMSE-Based Variable CSI Uncertainty: Consider the realistic MMSE estimator which has the error variance given by $\epsilon^{2}=\left(1+E_{s} / N_{0}\right)^{-1}[10]$. Thus, using (22), we attain the asymptotic SEP with MMSE CSI uncertainty as

$$
\begin{aligned}
\bar{P}_{s} & \approx \frac{43}{24}\left[\Psi\left(\frac{K}{N}+\frac{1}{2}\right)^{2}+\frac{\vartheta}{8 \rho^{2}}\left(\frac{K}{N}+1\right)^{2}\right]\left(\frac{1}{\gamma_{0}^{2}}\right) \\
& =\Theta\left(\left(c_{v} \gamma_{0}\right)^{-2}\right),
\end{aligned}
$$

where $\vartheta$ is given in (13) and the corresponding coding gain is

$$
c_{v}=\frac{1}{\sqrt{\frac{43}{24}\left[\Psi\left(\frac{K}{N}+\frac{1}{2}\right)^{2}+\frac{\vartheta}{8 \rho^{2}}\left(\frac{K}{N}+1\right)^{2}\right]}} .
$$

Notice that under MMSE CSI uncertainty, ReMO achieves the diversity order of two. However, comparing to the perfect CSI case, the proposed scheme suffers from a loss in the coding gain calculated by $10 \log \left(c_{p} / c_{v}\right)$, which is

$$
\Delta= \begin{cases}5 \log _{10}\left\{\frac{\frac{43}{24}\left[\Psi\left(1+\frac{N}{4}\right)^{2}+\frac{1}{8 \rho^{2}}\left(1+\frac{N}{4}\right)^{2}\right]}{\frac{43}{24}\left(\Psi+\frac{1}{8 \rho^{2}}\right)}\right\}, & \text { for } K=2 \\ 10 \log _{10}\left(1+\frac{N}{2 K}\right), & \text { for } K>2\end{cases}
$$

From (26), we can find that the coding gain distance $\Delta$ in $\mathrm{dB}$, gets larger as $K$ gets smaller.

\section{Simulation RESUlts AND DisCUSSION}

We now present numerical and simulation results to demonstrate the SEP and BER of ReMO in the presence of various CSI conditions. For comparison, the classical OFDM, MCIKOFDM [2], CI-OFDM-IM [13], and OFDM-IM-TD [14] with the ML detection are chosen as reference schemes. The peakto-average power ratio (PAPR) of ReMO is also discussed. 


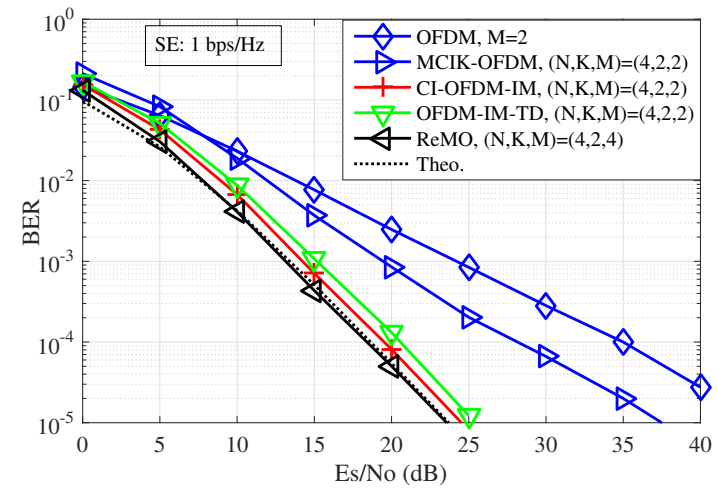

Fig. 2. BER comparison among classical OFDM with $M=2$, MCIK-OFDM, CI-OFDM-IM and OFDM-IM-TD with $(N, K, M)=(4,2,2)$, and ReMO with $(N, K, M)=(4,2,4)$, at the SE of $1 \mathrm{bps} / \mathrm{Hz}$.

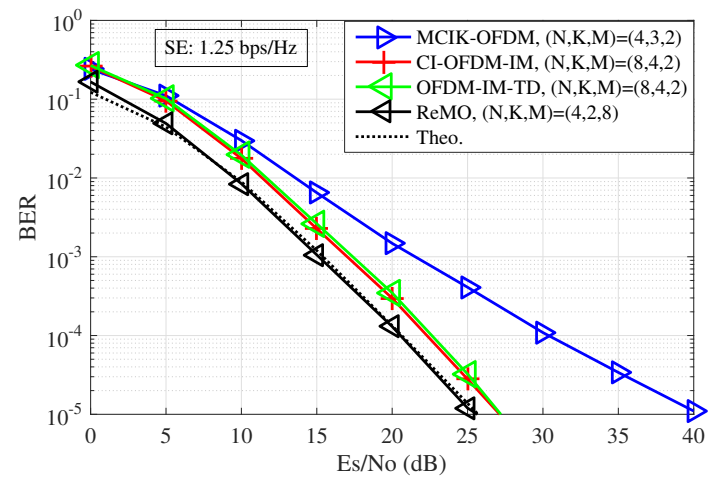

Fig. 3. BER comparison among MCIK-OFDM with $(N, K, M)=(4,3,2)$, CI-OFDM-IM and OFDM-IM-TD with $(N, K, M)=(8,4,2)$, and ReMO with $(N, K, M)=(4,2,4)$, at the SE of $1.25 \mathrm{bps} / \mathrm{Hz}$.

\section{A. ReMO under Perfect CSI}

Fig. 1 compares the average SEP of ReMO with four reference schemes at the SE of $1 \mathrm{bps} / \mathrm{Hz}$. As seen from Fig. 1, at the SEP of $10^{-4}$, the proposed scheme achieves significant SNR gains of more than $14 \mathrm{~dB}$ and $9 \mathrm{~dB}$ over OFDM and MCIK-OFDM, respectively. In addition, the SEP of our proposed scheme is close to that of CI-OFDM-IM, which is slightly better than OFDM-IM-TD. This is understandable since these three schemes attain the diversity order of two, which is twice larger than that of two classical schemes. Theoretical and asymptotic bounds are tight at almost SNRs, which validates the accuracy of expressions in (11) and (13).

Notice that the number of bits conveyed by index or $M$ ary symbols among different schemes are different. In the following, we would compare the proposed scheme with reference schemes in terms of the BER.

Fig. 2 depicts the BER comparison among schemes with the same parameters as Fig. 1. Unlike Fig. 1, the proposed scheme in Fig. 2 provides a better BER over CI-OFDM-IM and OFDM-IM-TD. Particularly, at the BER of $10^{-4}$, our scheme achieves SNR gains of $1 \mathrm{~dB}$ and $2 \mathrm{~dB}$ over CI-OFDMIM and OFDM-IM-TD, respectively.

The outstanding BER of ReMO can also be seen via Fig. 3 with the higher SE of $1.25 \mathrm{bps} / \mathrm{Hz}$. It is clear from Fig. 3 that our scheme offers the SNR gains of about $2 \mathrm{~dB}$ and $10 \mathrm{~dB}$ over

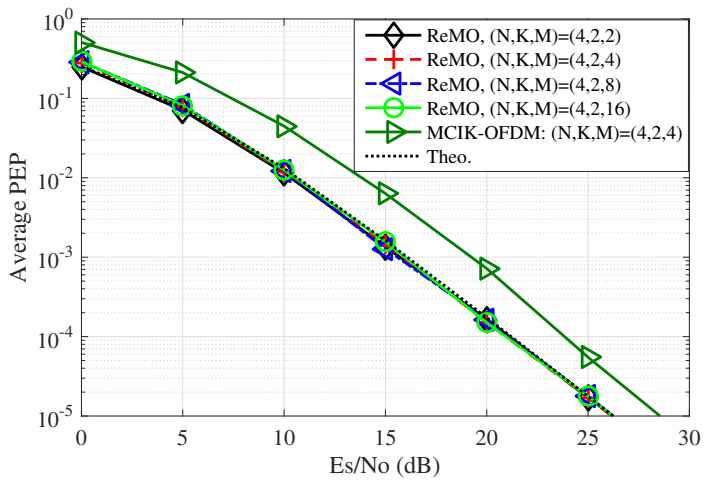

Fig. 4. Average PEP comparison between ReMO and MCIK-OFDM, when $N=4, K=2$, and $M=\{2,4,8,16\}$.

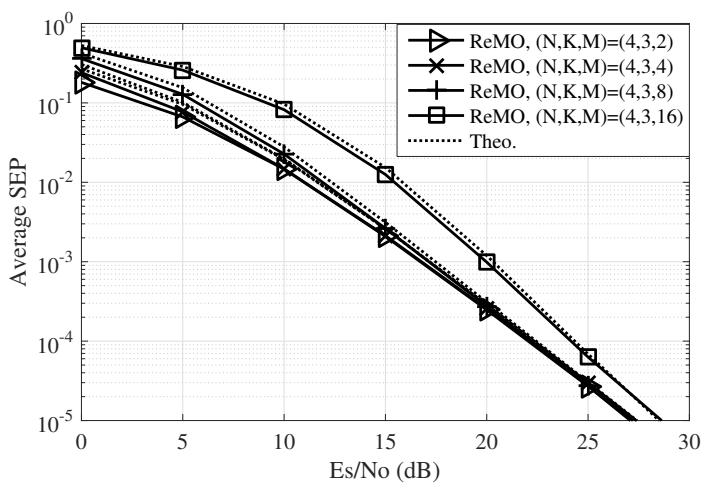

Fig. 5. Impact of $M$ on the SEP performance of ReMO when $N=4$, $K=3$, and $M=\{2,4,8,16\}$.

both CI-OFDM-IM and OFDM-IM-TD, and MCIK-OFDM, respectively. The two figures also validate the accuracy of the BER expression (12).

Fig. 4 depicts the behaviour of the average PEP for various $M$, and comparison with MCIK-OFDM. As observed from Fig. 4, the PEP remains unchanged when $M$ increases from 2 to 16 . This is because using the M-ary PSK, the conditional PEP in (3) is affected only by the energy per symbol, i.e., $\varphi E_{s}$, and not by the modulation size $M$. The PEP curves of two schemes have the same slope, indicating that they have the same diversity order. However, the proposed scheme achieves a better coding gain of about $2.5 \mathrm{~dB}$ over MCIK-OFDM at high SNRs. Fig. 3 also validates the tightness of the PEP expression in Theorem 1.

In Fig. 5, the impact of $M$ on the SEP with large $K$ is presented, when $N=4, K=3$, and $M=\{2,4,8,16\}$. It is shown that the average SEPs remain relatively unchanged as $M$ increases from 2 to 8, especially at high SNRs. Hence, the SEP mainly depends on the index detection errors rather than the $M$-ary symbol detection errors when $K>2$. This clearly validates Remark 2 . The considerable difference only appears when $M=16$.

Fig. 6 illustrates the impact of $K$ on the SEP when $N=5$, $M=\{4,16\}$ and $K=\{2,3,4\}$. As seen in Fig. 6, when $M$ is small, i.e., $M=4$, the system with smaller $K$ provides the better performance. Thus, $K=2$ is the best choice to minimizing the SEP. By contrast, as $M=16$, the system 


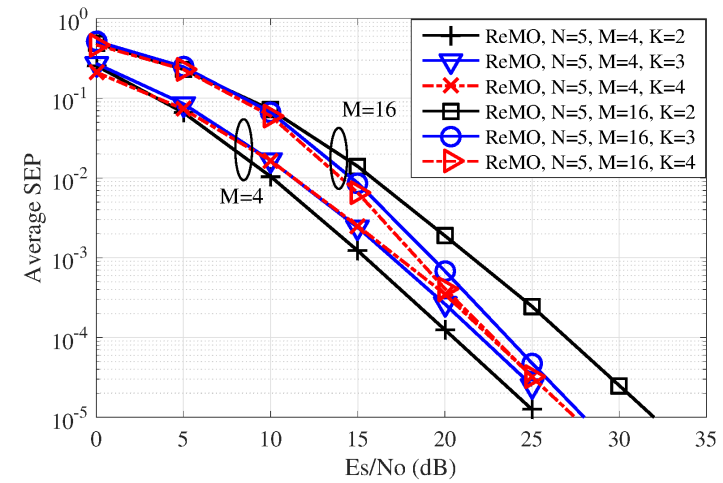

Fig. 6. Impact of $K$ on the SEP performance of ReMO when $N=5$, $K=\{2,3,4\}$, and $M=\{4,16\}$.

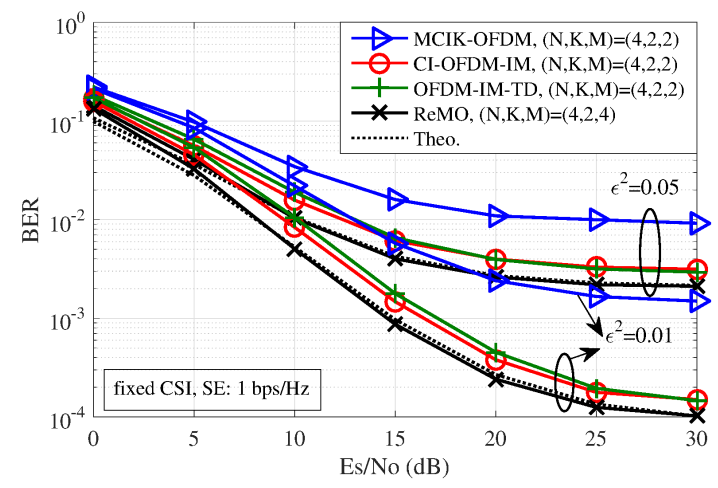

Fig. 7. BER comparison among MCIK-OFDM, CI-OFDM-IM and OFDMIM-TD with $(N, K, M)=(4,2,2)$, and ReMO with $(N, K, M)=(4,2,4)$, under fixed CSI with $\epsilon^{2}=\{0.05,0.01\}$, at the SE of $1 \mathrm{bps} / \mathrm{Hz}$.

achieves the better SEP when $K$ gets larger, i.e., $K=4$ offers the best SEP. As a result, Remark 3 is validated.

\section{B. ReMO under Uncertain CSI}

Fig. 7 compares the BER among proposed and existing schemes, under fixed CSI conditions with $\epsilon^{2}=\{0.05,0.01\}$, at the SE of $1 \mathrm{bps} / \mathrm{Hz}$. As seen from Fig. 7, there are error floors caused by fixed $\epsilon^{2}(>0)$, which get higher as $\epsilon^{2}$ increases. Interestingly, under various $\epsilon^{2}>0$, the proposed scheme still significantly outperforms the reference schemes. For example, at the BER of $10^{-2}$ and $\epsilon^{2}=0.05$, our scheme provides SNR gains of about $2.5 \mathrm{~dB}, 3 \mathrm{~dB}$ and more than $10 \mathrm{~dB}$ over CIOFDM-IM, OFDM-IM-TD and MCIK-OFDM, respectively.

In Fig. 8, we present the impact of MMSE-based CSI uncertainty on the SEP when $N=4, K=\{2,3\}, M=\{4,8\}$. It can be seen that the proposed scheme with $K=3$ suffers from the performance loss of slightly less than $2.5 \mathrm{~dB}$ at high SNRs, compared to the perfect CSI case. This confirms the accuracy of the theoretical coding gain loss in (26) with $\Delta=2.2 \mathrm{~dB}$. Furthermore, when $K$ is smaller, i.e., $K=2$, the coding gain loss caused by MMSE CSI uncertainty becomes larger, which is about $4 \mathrm{~dB}$ in Fig. 8.

Fig. 9 depicts the BER comparison among ReMO and reference schemes under MMSE CSI uncertainty, at the SE of 1.5 bps/Hz. Notice that the proposed scheme employs 16-QAM rather than 16-PSK to achieve the better BER at large $M$. As

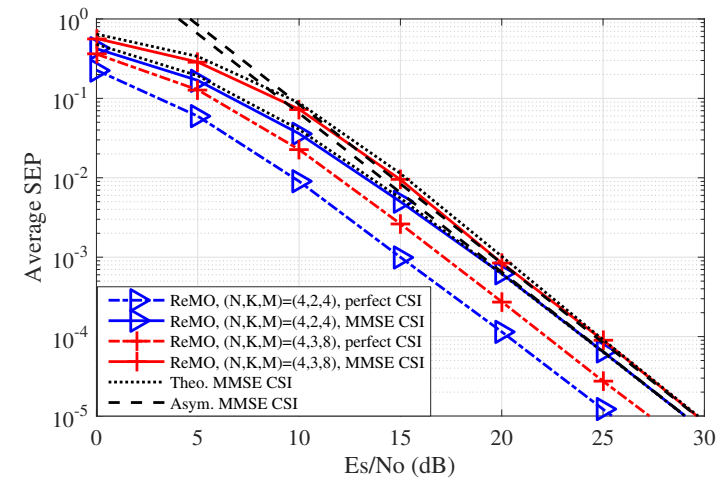

Fig. 8. Impact of MMSE-based CSI uncertainty on the average SEP of ReMO when $N=4, K=\{2,3\}, M=\{4,8\}$.

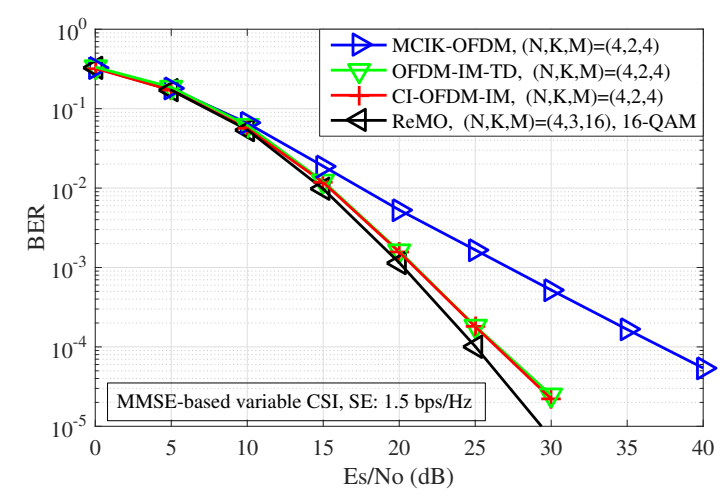

Fig. 9. BER comparison among MCIK-OFDM, CI-OFDM-IM and OFDMIM-TD with $(N, K, M)=(4,2,4)$, and ReMO with $(N, K, M)=$ $(4,3,16)$, under MMSE-based CSI uncertainty, at the SE of $1.5 \mathrm{bps} / \mathrm{Hz}$.

observed from Fig. 9, our proposed scheme is superior to the reference schemes under MMSE CSI uncertainty, especially at high SNRs. In particular, at the BER of $10^{-4}$, ReMO provides SNR gains of $1.5 \mathrm{~dB}$ over CI-OFDM-IM and OFDM-IM-TD, and $12 \mathrm{~dB}$ over MCIK-OFDM.

In summary, it is noteworthy that ReMO without using any additional techniques such as CI, I/Q, LCP and multiple constellations, still outperforms reference schemes under both perfect and imperfect CSI, at the low data rate $(\leq 1.5 \mathrm{bps} / \mathrm{Hz})$. However, for higher data rate $(\geq 2 \mathrm{bps} / \mathrm{Hz})$, the proposed scheme requires the larger $M$-ary modulation size, which leads to the degradation of error performance. Hence, the repetition coded MCIK-OFDM with its simple but effective design can be suitable for various MTC applications that require lowthroughput and low-complexity, but very high reliability.

\section{Discussion about PAPR}

Employing the repetition code, ReMO may suffer from a notable increase in the PAPR compared to the classical MCIK-OFDM and OFDM, as shown in Fig. 10. This issue becomes more severe when $K$ gets larger. However, we can employ the Zadoff-Chu (ZC) sequence [24] of the length $N_{c}$ to substantially reduce the PAPR of ReMO, as seen via Fig. 10, where we recall that $N_{c}$ is the total number of sub- 


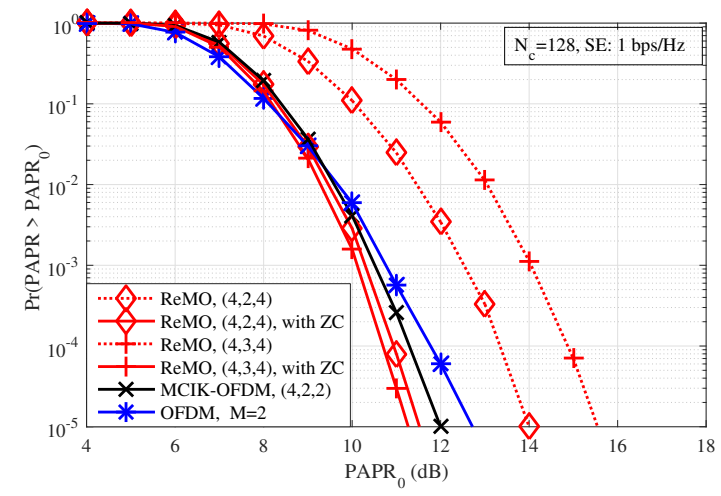

Fig. 10. PAPR comparison among classical OFDM, MCIK-OFDM, and ReMO with or without using the Zadoff-Chu sequence for PAPR reduction. The configurations of ReMO and MCIK-OFDM are presented by $(N, K, M)$.

carriers from $G$ clusters. Particularly, the ZC sequence of $\mathbf{z}=\left[z_{1}, \ldots, z_{N_{c}}\right]$ is defined by

$$
z_{n}=\left\{\begin{array}{ll}
e^{-\frac{j 2 \pi m}{N_{c}}\left(\frac{n^{2}}{2}+q n\right)} & \text { for } N_{c} \text { even } \\
e^{-\frac{j 2 \pi m}{N_{c}}\left[\frac{n(n+1)}{2}+q n\right]} & \text { for } N_{c} \text { odd }
\end{array},\right.
$$

where $m$ is any integer relatively prime to $N_{c}, q$ is any integer, and $n=1, \ldots, N_{c}$. The use of the $\mathrm{ZC}$ in the proposed scheme is not complicated as follows. At the transmitter, signal vectors $\mathbf{x}$ of all $G$ clusters are multiplied by $\mathbf{z}$ before entering the inverse fast Fourier transform. Then, the receiver simply utilizes $\mathbf{z}^{-1}$ to suppress the effect of the $\mathrm{ZC}$ on the received signal prior to the detection process.

Fig. 10 clearly validates the effectiveness of using the $\mathrm{ZC}$ for the PAPR reduction in the ReMO. Especially, when the $\mathrm{ZC}$ is employed, the proposed ReMO always achieves a better PAPR than the classical MCIK-OFDM and OFDM, even when $K$ increases. Meanwhile, notice that the $\mathrm{ZC}$ method does not help classical schemes to improve the PAPR since their data symbols carried on active sub-carriers are already distinct.

\section{CONCLUSIONS}

We have discovered potentials of the repeated MCIKOFDM scheme, which can provide a significantly improved error performance over existing schemes. Specifically, by repeatedly transmitting the same $M$-ary symbol over all active sub-carriers and simultaneously using their indices as an additional dimension, the proposed scheme is capable of exploiting both the frequency diversity gain and the index coding gain. This allows to achieve a notable improvement in the transmit diversity compared to current schemes, including CI-OFDMIM, OFDM-IM-TD, classical OFDM-IM and OFDM. For performance analysis, we derived tight, closed-form expressions for the PEP, SEP and BER, under both perfect and imperfect CSI. This provided an insight into the achievable performance gains and impacts of system parameters and various CSI conditions on the performance. Simulation results show that the proposed scheme not only has simpler transceiver design, but also outperforms benchmark schemes under a range of uncertain CSI, even at the same data rate.

\section{APPENDIX}

\section{A. Proof of Theorem 1}

The average PEP can be given, by taking the expectation of (7), as

$$
\begin{aligned}
\bar{P}_{I} & \approx \frac{1}{m_{1}} \sum_{i=1}^{m_{1}} \sum_{j \in \Omega_{i}} \mathbb{E}_{\gamma_{\Sigma}}\left\{Q\left[\sqrt{\frac{\gamma_{\Sigma}}{2}}\right]\right\} \\
& \approx \mathbb{E}_{\gamma_{\Sigma}}\left\{\frac{\sum_{i=1}^{m_{1}} \eta_{i}}{m_{1}}\left(\frac{1}{12} e^{-\frac{\gamma_{\Sigma}}{4}}+\frac{1}{4} e^{-\frac{\gamma_{\Sigma}}{3}}\right)\right\},
\end{aligned}
$$

where $\gamma_{\Sigma}=\gamma_{\alpha}+\gamma_{\tilde{\alpha}}$ and we use the approximation of Qfunction as

$$
Q(x) \approx \frac{1}{12} e^{-x^{2} / 2}+\frac{1}{4} e^{-2 x^{2} / 3} .
$$

Due to the definition of the moment generating function (MGF) [23], i.e., $\mathcal{M}_{\gamma}(z)=\mathbb{E}_{\gamma}\left\{e^{-z \gamma}\right\}=(1-\bar{\gamma} z)^{-1}$ for the Rayleigh fading channel, the MGF of $\gamma_{\Sigma}$ can be calculated as $\mathcal{M}_{\gamma_{\Sigma}}(z)=\mathcal{M}_{\gamma}^{2}(z)=(1-\bar{\gamma} z)^{-2}$. Denote $\eta=\sum_{i=1}^{m_{1}} \eta_{i}$ and $\Psi=\eta / m_{1}$. Finally, based on the MGF approach, the approximate average PEP in (28) can be attained as

$$
\begin{aligned}
\bar{P}_{I} & \approx \frac{\Psi}{12}\left[\mathcal{M}_{\gamma_{\Sigma}}\left(e^{-\frac{\gamma_{\Sigma}}{4}}\right)+3 \mathcal{M}_{\gamma_{\Sigma}}\left(e^{-\frac{\gamma_{\Sigma}}{3}}\right)\right] \\
& \approx \frac{\Psi}{12}\left[\frac{16}{(4+\bar{\gamma})^{2}}+\frac{27}{(3+\bar{\gamma})^{2}}\right] .
\end{aligned}
$$

\section{B. Proof of Theorem 2}

Since the $M$-ary symbol is carried on $K$ different sub-channels, the ML detects this symbol with an instantaneous SNR of $\gamma_{\Sigma_{\alpha}}=\sum_{k=1}^{K} \gamma_{\alpha_{k}}$, where $\alpha_{k} \in$ $\theta_{i}$. Thus, $P_{M}$ in (9) can be approximated by $P_{M} \approx$ $2 Q\left(\sqrt{2 \gamma_{\Sigma_{\alpha}}} \sin (\pi / M)\right)$ [25]. Using (28), we can represent $P_{M} \approx\left(e^{-\rho \gamma_{\alpha}}+3 e^{-4 \rho \gamma_{\Sigma_{\alpha}} / 3}\right) / 6$, where $\rho=\sin ^{2}(\pi / M)$. Here, similar to the proof of Theorem 1 and notice that $\mathcal{M}_{\gamma_{\Sigma_{\alpha}}}(z)=\mathcal{M}_{\gamma}^{K}(z)=(1-\bar{\gamma} z)^{-K}$, the average of $P_{M}$ can be attained as

$$
\bar{P}_{M} \approx \frac{1}{6}\left[(1+\rho \bar{\gamma})^{-K}+3(1+4 \rho \bar{\gamma} / 3)^{-K}\right] .
$$

Finally, using (8), (10), and (30), we obtain (11).

\section{Proof of Theorem 3}

Denote $\tilde{N}_{0}=1+\bar{\gamma} \epsilon^{2} / 2$ and $\hat{\gamma}_{\Sigma}=\hat{\gamma}_{\alpha}+\hat{\gamma}_{\tilde{\alpha}}$. Using the approximation of Q-function in (29), we obtain from (19) that

$$
P_{I} \approx \frac{1}{m_{1}} \sum_{i=1}^{m_{1}} \sum_{j \in \Omega_{i}}\left(\frac{1}{12} e^{-\hat{\gamma}_{\Sigma} / 4 \widetilde{N}_{0}}+\frac{1}{4} e^{-\hat{\gamma}_{\Sigma} / 3 \widetilde{N}_{0}}\right) .
$$

Due to system model, the MGF of $\hat{\gamma}_{\Sigma}$ is given by $\mathcal{M}_{\hat{\gamma}_{\Sigma}}(s)=\mathcal{M}_{\hat{\gamma}}^{2}(s)=\left[1-\bar{\gamma}\left(1-\epsilon^{2}\right) s\right]^{-2}$. Thus, applying the MGF approach for (31), the average PEP under CSI uncertainty can be obtain as (20). This concludes the proof. 


\section{Proof of Lemma 1}

Under imperfect CSI (16), the received signal at active subcarrier is given by $y(\alpha)=\hat{h}(\alpha) s+\tilde{n}(\alpha)$, where $\tilde{n}(\alpha)=$ $e(\alpha) s+n(\alpha) \sim \mathcal{C N}\left(0,\left(\bar{\gamma} \epsilon^{2}+1\right) N_{0}\right)$. Since the symbol $s$ is the same for all active sub-carriers $\alpha \in \theta$, the ML will detect $s$ with an instantaneous SNR of $\hat{\gamma}_{\Sigma_{\alpha}}=\sum_{\alpha \in \theta} \hat{\gamma}_{\alpha} /\left(1+\bar{\gamma} \epsilon^{2}\right)$, where $\hat{\gamma}_{\alpha}=\bar{\gamma}|\hat{h}(\alpha)|^{2}$. Similar to the proof of Theorem 2,

$$
P_{M} \approx \frac{1}{6}\left(e^{-\rho \hat{\gamma}_{\Sigma_{\alpha}}}+3 e^{-4 \rho \hat{\gamma}_{\Sigma_{\alpha}} / 3}\right)
$$

where $\rho=\sin ^{2}(\pi / M)$. Note that the MGF of $\hat{\gamma}_{\Sigma_{\alpha}}$ can be obtained by $\mathcal{M}_{\hat{\gamma}_{\Sigma_{\alpha}}}(s)=\mathcal{M}_{\hat{\gamma}}^{K}(s)=\left[1-\bar{\gamma}\left(1-\epsilon^{2}\right) s\right]^{-K}$.

Finally, utilizing the MGF approach in (32), $\bar{P}_{M}$ can be obtained as in (21). This concludes the proof.

\section{REFERENCES}

[1] R. Abu-alhiga and H. Haas, "Subcarrier-index modulation OFDM," in Proc. IEEE Pers., Indoor., Mobile Radio Commun., Sept 2009, pp. 177181 .

[2] E. Basar, U. Aygolu, E. Panayirci, and H. V. Poor, "Orthogonal frequency division multiplexing with index modulation,' IEEE Trans. Signal Process., vol. 61, no. 22, pp. 5536-5549, Nov 2013.

[3] C. Bockelmann, N. Pratas, H. Nikopour, K. Au, T. Svensson, C. Stefanovic, P. Popovski, and A. Dekorsy, "Massive machine-type communications in 5g: physical and MAC-layer solutions," IEEE Commun. Mag., vol. 54, no. 9, pp. 59-65, September 2016.

[4] E. Basar, M. Wen, R. Mesleh, M. D. Renzo, Y. Xiao, and H. Haas, "Index modulation techniques for next-generation wireless networks," IEEE Access, vol. 5, pp. 16693-16746, 2017.

[5] Y. Ko, "A tight upper bound on bit error rate of joint OFDM and multicarrier index keying," IEEE Commun. Lett., vol. 18, no. 10, pp. 1763 1766, Oct 2014.

[6] M. Wen, X. Cheng, M. Ma, B. Jiao, and H. V. Poor, "On the achievable rate of OFDM with index modulation," IEEE Trans. Signal Process., vol. 64, no. 8, pp. 1919-1932, April 2016.

[7] B. Zheng, F. Chen, M. Wen, F. Ji, H. Yu, and Y. Liu, "Lowcomplexity ML detector and performance analysis for OFDM with in-phase/quadrature index modulation," IEEE Commun. Lett., vol. 19, no. 11, pp. 1893-1896, Nov 2015.

[8] R. Fan, Y. J. Yu, and Y. L. Guan, "Generalization of orthogonal frequency division multiplexing with index modulation," IEEE Trans. Wireless Commun., vol. 14, no. 10, pp. 5350-5359, Oct 2015.

[9] T. Mao, Z. Wang, Q. Wang, S. Chen, and L. Hanzo, "Dual-mode index modulation aided OFDM," IEEE Access, vol. 5, pp. 50-60, 2017.

[10] T. V. Luong and Y. Ko, "Impact of CSI uncertainty on MCIK-OFDM: tight, closed-form symbol error probability analysis," IEEE Trans. Veh. Technol., vol. 67, no. 2, pp. 1272 - 1279, Feb 2018.

[11] _ "A tight bound on BER of MCIK-OFDM with greedy detection and imperfect CSI," IEEE Commun. Lett., vol. 21, no. 12, pp. $2594-$ 2597, Dec 2017.

[12] Y. Xiao, S. Wang, L. Dan, X. Lei, P. Yang, and W. Xiang, "OFDM with interleaved subcarrier-index modulation,” IEEE Commun. Lett., vol. 18, no. 8, pp. 1447-1450, Aug 2014.

[13] E. Basar, "OFDM with index modulation using coordinate interleaving," IEEE Wireless Commun. Lett., vol. 4, no. 4, pp. 381-384, Aug 2015.

[14] J. Zheng and R. Chen, "Achieving transmit diversity in OFDM-IM by utilizing multiple signal constellations," IEEE Access, vol. 5, no. 99, pp. 8978 - 8988, 2017.

[15] M. Wen, B. Ye, E. Basar, Q. Li, and F. Ji, "Enhanced orthogonal frequency division multiplexing with index modulation," IEEE Trans. Wireless Commun., vol. 16, no. 7, pp. 4786 - 4801, July 2017.

[16] J. Choi, "Coded OFDM-IM with transmit diversity," IEEE Trans. Commun., vol. 65, no. 7, pp. 3164-3171, July 2017.

[17] E. Basar, "On multiple-input multiple-output OFDM with index modulation for next generation wireless networks," IEEE Trans. Signal Process., vol. 64, no. 15, pp. 3868-3878, Aug 2016.

[18] B. Zheng, M. Wen, E. Basar, and F. Chen, "Multiple-input multipleoutput OFDM with index modulation: Low-complexity detector design," IEEE Trans. Signal Process., vol. 65, no. 11, pp. 2758-2772, June 2017.
[19] J. Crawford, E. Chatziantoniou, and Y. Ko, "On the SEP analysis of OFDM index modulation with hybrid low complexity greedy detection and diversity reception," IEEE Trans. Veh. Technol., vol. 66, no. 9, pp. 8103-8118, Sept 2017.

[20] T. V. Luong and Y. Ko, "The BER analysis of MRC-aided greedy detection for OFDM-IM in presence of uncertain CSI," IEEE Wireless Commun. Lett., to be published.

[21] L. Wang, Z. Chen, Z. Gong, and M. Wu, "Space-frequency coded index modulation with linear-complexity maximum likelihood receiver in the MIMO-OFDM system," IEEE Signal. Proc. Let., vol. 23, no. 10, pp. 1439-1443, Oct 2016.

[22] T. V. Luong and Y. Ko, "A closed-form symbol error probability for MCIK-OFDM with frequency diversity," in Proc. IEEE SPAWC, Jul 2017, pp. 1-5.

[23] M. K. Simon and M.-S. Alouini, Digital communication over fading channels, ser. Wiley series in telecommunications and signal processing. Hoboken, N.J. Wiley-Interscience, 2005.

[24] D. Chu, "Polyphase codes with good periodic correlation properties," IEEE Trans. Inf. Theory, vol. 18, no. 4, pp. 531-532, July 1972.

[25] J. Proakis, Digital Communications. McGraw-Hill, 2001. 